\title{
Volumetric change of the latissimus dorsi muscle after postoperative radiotherapy in immediate breast reconstruction with an extended latissimus dorsi musculocutaneous flap
}

\author{
Tae Seo Park ${ }^{1}$, Jung Yeol Seo ${ }^{1}$, Anvar S. Razzokov' ${ }^{2}$ June Seok Choi ${ }^{1}$, Min Wook Kim ${ }^{1}$, \\ Jae Woo Lee ${ }^{1}$, Hyun Yeol Kim ${ }^{3}$, Youn Joo Jung ${ }^{3}$, Ki Seok Choo ${ }^{4}$, Kyeong Ho Song ${ }^{5}$, Su Bong Nam ${ }^{1}$ \\ ${ }^{1}$ Department of Plastic and Reconstructive Surgery, Pusan National University School of Medicine, Yangsan, Korea; ${ }^{2}$ Medion Hospital, \\ Tashkent, Uzbekistan; ${ }^{3}$ Breast Surgery Center, Pusan National University Yangsan Hospital, Yangsan; ${ }^{4}$ Department of Radiology, Pusan \\ National University School of Medicine, Yangsan; ${ }^{5}$ Four Seasons Plastic Surgery Clinic, Busan, Korea
}

Background This study aimed to determine the magnitude of volume reduction of the latissimus dorsi (LD) muscle after treatment using only postoperative radiotherapy (PORTx) in patients who underwent immediate breast reconstruction using an extended LD musculocutaneous (eLDMC) flap after partial mastectomy.

Methods We retrospectively reviewed 28 patients who underwent partial mastectomy and an eLDMC flap, received only PORTX, and underwent chest computed tomography (CT) 7 to 10 days after surgery and $18 \pm 4$ months after the end of radiotherapy, from March 2011 to June 2016. The motor nerve to the LD was resected in all patients. One plastic surgeon performed the procedures, and the follow-up period was at least 36 months (mean, 46.6 months). The author obtained LD measurements from axial CT views, and the measurements were verified by an experienced radiologist. The threshold for statistical significance was set at $\mathrm{P}<0.05$.

Results A statistically significant decrease in the LD volume was found after the end of PORTx (range, 61.19\%-80.82\%; mean, 69.04\%) in comparison to the measurements obtained 7 to 10 days postoperatively $(\mathrm{P}<0.05)$. All cases were observed clinically for over 3 years. Conclusions The size of an eLDMC flap should be determined considering an average LD reduction of $69 \%$ after PORTx. Particular care should be taken in determining the size of an eLDMC flap if the LD is thick or if it occupies a large portion of the flap.

Keywords Mammaplasty / Superficial back muscles / Myocutaneous flap
Correspondences: Kyeong Ho Song Four Seasons Plastic Surgery Clinic, 12 Bujeon-ro 66beon-gil, Busanjingu, Busan 47287, Korea Tel: +82-51-806-7979

E-mail: tonyseo85@gmail.com

Su Bong Nam

Department of Plastic and Reconstructive Surgery, Pusan National University School of Medicine, 49 Busandaehak-ro, Mulgeum-eup, Yangsan 50612, Korea Tel: +82-55-360-1439

Fax: +82-55-360-1684

E-mail: Subong71@hanmail.net

This study was supported by a grant of the clinical research fund of Pusan National University Yangsan Hospital in 2018.

Received: September 26, 2019 • Revised: November 29, 2019 • Accepted: December 23, 2019

pISSN: 2234-6163 • elSSN: 2234-6171 • https://doi.org/10.5999/aps.2019.01361 • Arch Plast Surg 2020;47:135-139

\section{INTRODUCTION}

The extended latissimus dorsi musculocutaneous (eLDMC) flap is one of the most commonly used methods for breast-conserving surgery (BCS) performed immediately after partial mastectomy [1-3]. 
After partial mastectomy, most patients should undergo radiotherapy. Therefore, consideration should be given to the effects of radiotherapy on the flap, and reconstruction should be carried out with due consideration of the breast volume reduction due to atrophy of the latissimus dorsi (LD) muscle.

In particular, resection of the motor nerve of the $\mathrm{LD}$ during surgery, which is performed in order to prevent synkinetic muscle movement, exacerbates the subsequent atrophy of the LD muscle. In addition, shrinkage of the flap due to radiotherapy makes it difficult to achieve symmetry of the breasts.

The purpose of this study was to evaluate the magnitude of volume reduction of the $\mathrm{LD}$ after treatment using only postoperative radiotherapy (PORTx) in patients who underwent immediate breast reconstruction with an eLDMC flap.

\section{METHODS}

Twenty-eight patients were selected who received partial mastectomy at our hospital and then underwent immediate breast reconstruction with an eLDMC flap performed. Follow-up computed tomography (CT) scans (Discovery CT $750 \mathrm{HD}$; GE Medical Systems, Milwaukee, WI, USA) using the same protocol were taken 7 to 10 days after the operation and $18 \pm 4$ months after the end of PORTx. PORTx was performed at our hospital, with a total of 33 fractions and 6,040 Gy (27 cases) or a total of 35 fractions and 6,500 Gy (1 case) (CLINAC IX; Varian Medical Systems, Inc., Palo Alto, CA, USA).

The dissection of the eLDMC flap was performed according to the standard method, and the lumbar fat pad was not included to reduce the risk of donor site seroma.

The procedures were performed by a single breast reconstructive surgeon. All patients underwent resection of the motor nerve of the LD and received over 3 years of postoperative follow-up.
The breast reconstructive surgeon obtained measurements of the LD using the picture archiving and communications system (PACS) from the axial view of chest CT scans, and the measurements were verified by an experienced professor from the department of radiology who specialized in breast imaging. In

\begin{tabular}{|c|c|c|c|}
\hline Case & $\begin{array}{c}\text { 7-10 Days } \\
\text { postoperatively } \\
\left(\mathrm{mm}^{3}\right)\end{array}$ & $\begin{array}{l}\text { 14-22 Months } \\
\text { after radiotherapy } \\
\left(\mathrm{mm}^{3}\right)\end{array}$ & $\begin{array}{c}\text { Volume } \\
\text { decrease }(\%)\end{array}$ \\
\hline 1 & $74,118.4$ & $26,592.6$ & 64.12 \\
\hline 2 & $64,040.7$ & $22,880.8$ & 64.27 \\
\hline 3 & $137,269.3$ & $50,920.8$ & 62.90 \\
\hline 4 & $115,077.1$ & $44,660.3$ & 61.19 \\
\hline 5 & $97,609.9$ & $29,574.7$ & 69.70 \\
\hline 6 & $86,025.9$ & $30,314.6$ & 64.76 \\
\hline 7 & $91,912.9$ & $25,541.1$ & 72.21 \\
\hline 8 & $98,552.5$ & $35,186.1$ & 64.30 \\
\hline 9 & $107,405.0$ & $31,806.2$ & 70.39 \\
\hline 10 & $104,104.1$ & $29,126.7$ & 72.02 \\
\hline 11 & $114,065.9$ & $21,874.9$ & 80.82 \\
\hline 12 & $102,496.9$ & $25,938.1$ & 74.69 \\
\hline 13 & $103,351.0$ & $26,948.9$ & 73.92 \\
\hline 14 & $134,889.7$ & $42,426.9$ & 68.55 \\
\hline 15 & $110,506.6$ & $32,651.7$ & 70.45 \\
\hline 16 & $113,931.1$ & $39,381.3$ & 65.43 \\
\hline 17 & $122,856.5$ & $42,809.2$ & 65.16 \\
\hline 18 & $173,854.9$ & $59,712.4$ & 65.65 \\
\hline 19 & $154,781.2$ & $43,371.6$ & 71.98 \\
\hline 20 & $142,440.2$ & $43,781.6$ & 69.26 \\
\hline 21 & $101,695.4$ & $31,499.7$ & 69.03 \\
\hline 22 & $137,998.7$ & $37,566.6$ & 72.78 \\
\hline 23 & $97,359.5$ & $32,170.6$ & 66.96 \\
\hline 24 & $115,636.0$ & $27,885.5$ & 75.89 \\
\hline 25 & $109,088.8$ & $37,646.4$ & 65.49 \\
\hline 26 & $95,630.0$ & $21,612.4$ & 77.40 \\
\hline 27 & $166,511.9$ & $51,531.7$ & 69.05 \\
\hline 28 & $111,202.4$ & $39,255.2$ & 64.70 \\
\hline Average & & & 69.04 \\
\hline
\end{tabular}

\section{Fig. 1. Chest computed tomography, axial view}

(A) Seven days postoperatively (area of latissimus dorsi [LD] muscle, 2,652.07 mm²). (B) Eighteen months after postoperative radiotherapy (area of LD muscle, 1,123.29 $\mathrm{mm}^{2}$ ).
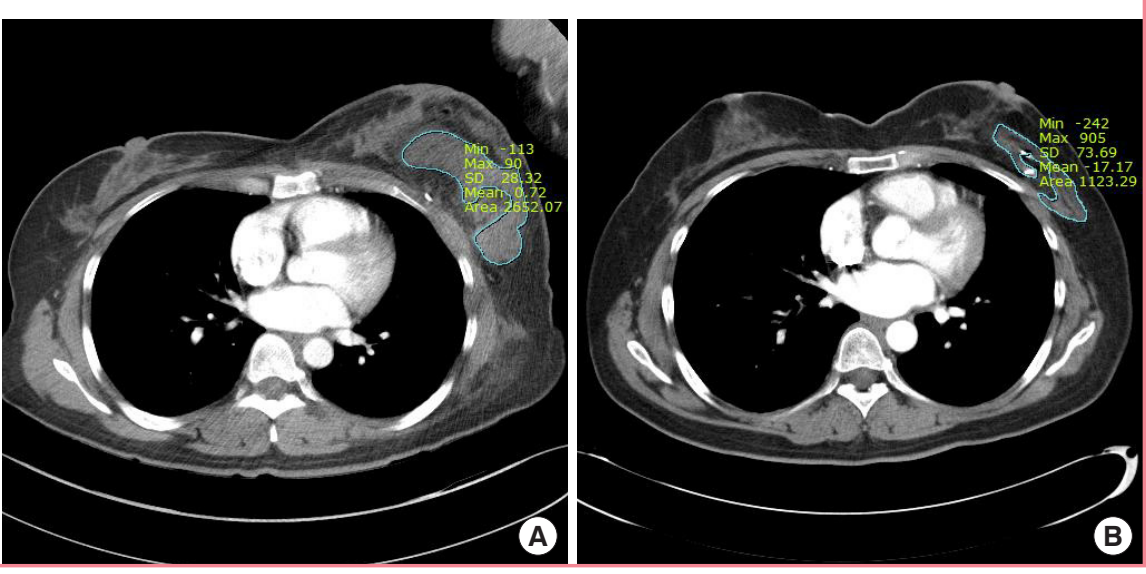
each CT image slice, the area of the LD muscle was marked, and the total volume was calculated by multiplying the total measured area by the thickness (in millimeters) of the CT slices (Fig. 1). The volume measurements were analyzed statistically using SPSS version 16.0 (SPSS Inc., Chicago, IL, USA), with $\mathrm{P}<0.05$ as the threshold for statistical significance.

\section{RESULTS}

The difference in the LD volume between 7 and 10 days immediately after the operation and $18 \pm 4$ months after the end of PORTx was statistically significant $(\mathrm{P}<0.05)$. The average volume decrease was $69.04 \%$, with a range of $61.19 \%$ to $80.82 \%$ (Table 1).

Clinically, over the course of more than 3 years of follow-up, both the surgeon and the patient were satisfied in 26 of the 28 cases. In the other two cases, one of the patients underwent bilateral breast augmentation because of personal preference, and the other patient was unsatisfied with the contracture deformity and received secondary correction through excision, release of the scar contracture, and implant insertion.

Fig. 2 presents a preoperative image of one of the cases, followed by a 1-month postoperative photograph (Fig. 3), and a photograph taken 3 years after the end of PORTx (Fig. 4).

\section{DISCUSSION}

The statistics of the Korean Central Cancer Registry indicate that 22,550 new cases of breast cancer and 2,353 deaths were reported in 2015, making breast cancer the second most common type of cancer in women (following thyroid cancer) [4]. BCS is frequently performed (62.3\%), but the proportion of mastectomies has been gradually increasing in recent years, from $32.3 \%$ in 2014 to $36.1 \%$ in 2015 [5]. Since BCS is generally performed in combination with PORTx, surgical reconstruction after BCS is usually difficult because of post-irradiation fibrosis, and the

\section{Fig. 2. Preoperative pictures of a patient}

The patient was 51 years old and had right breast cancer (T1NOM0). Her body mass index was $28 \mathrm{~kg} / \mathrm{m}^{2}$. The tumor was excised, and the mass of the excised tissue was 167 g. The extended latissimus dorsi musculocutaneous flap weighed 320 g. (A) Frontal view, (B) profile view, and (C) side view.
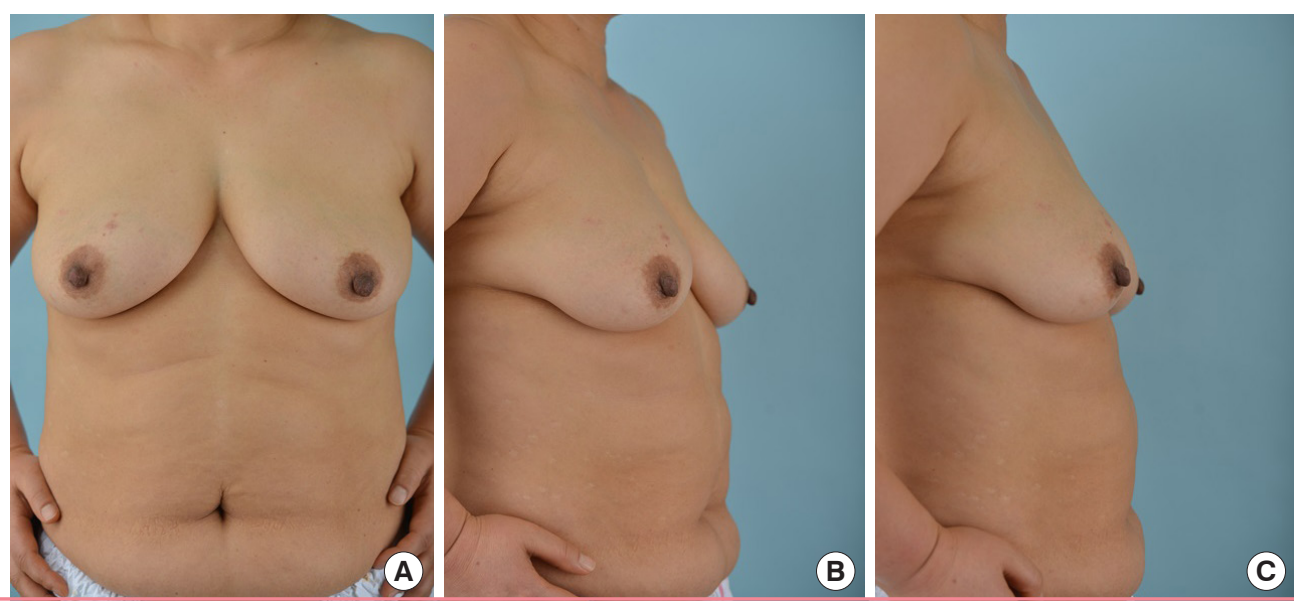

Fig. 3. One-month postoperative photographs (before radiotherapy)

(A) Frontal view, (B) profile view, and $(C)$ side view.
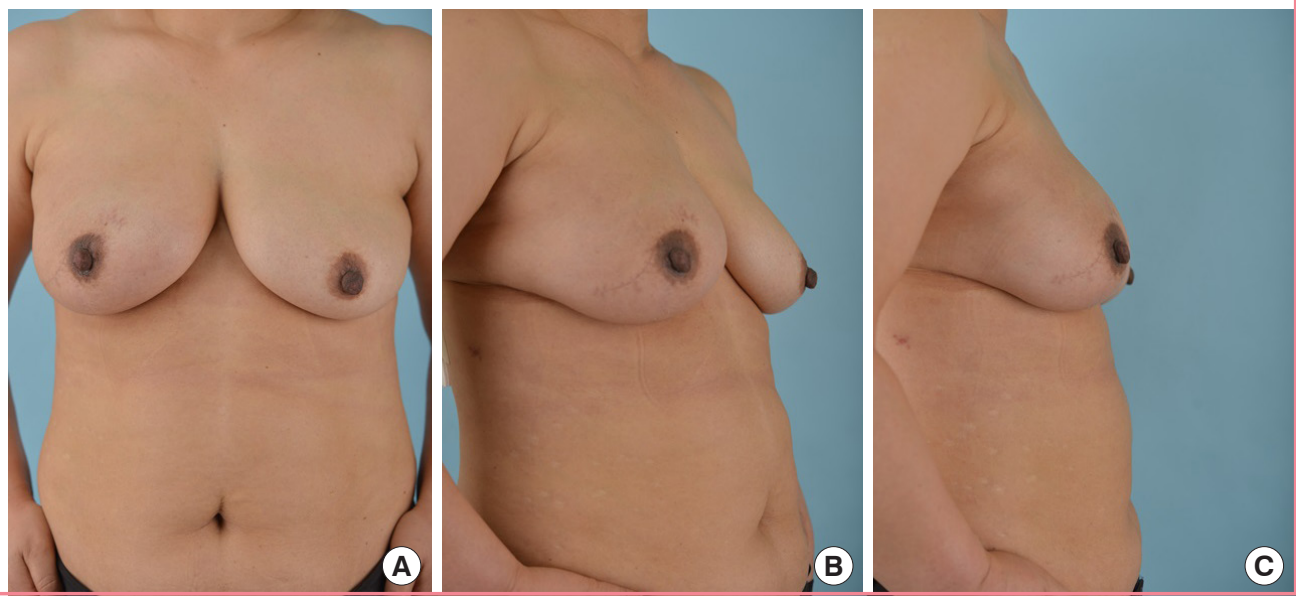
Fig. 4. Photographs taken 3 years after radiotherapy

(A) Frontal view, (B) profile view, and $(\mathrm{C})$ side view.
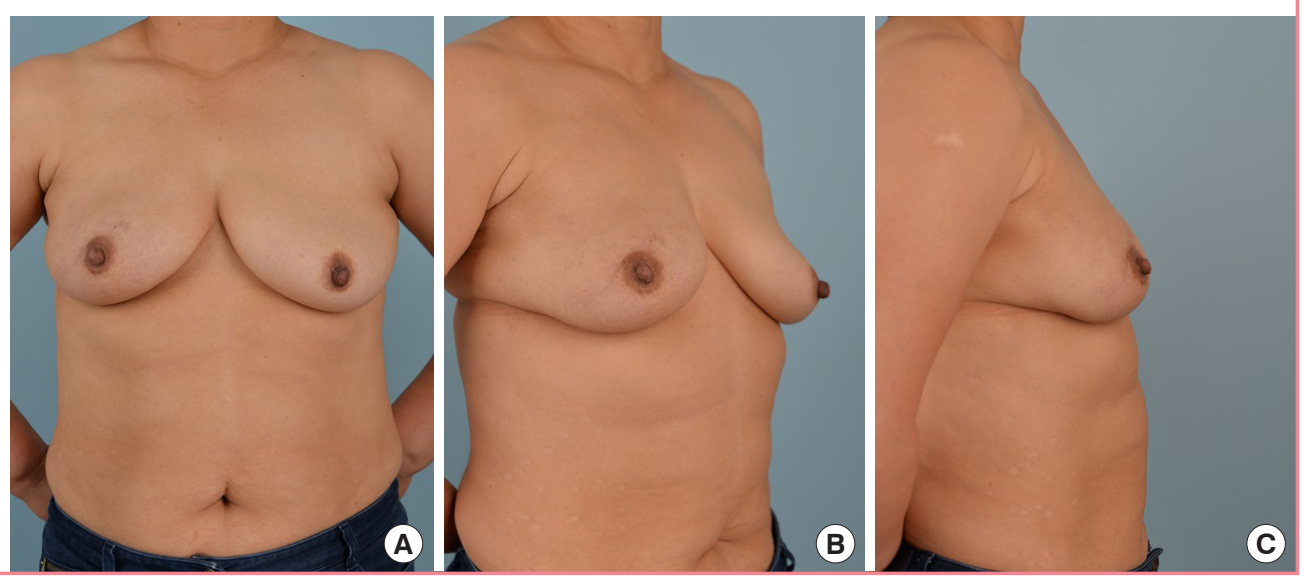

aesthetic results are often unpredictable [6].

Despite the safety and reliability of the eLDMC flap, it is often inevitable for the skin paddle to be exposed to the skin surface when correcting nipple-areola complex malposition, leaving conspicuous, patchwork-like scars on the breast. A critical advantage of the eLDMC flap is that it does not require microsurgical techniques and thereby allows patients to recover more quickly. Coupled with this relative technical ease is the modest frequency and magnitude of short-term complications [7]. Moreover, the eLDMC flap is relatively resistant to the higher rate of complications seen in obese patients undergoing prosthetic and abdominal-based reconstruction $[8,9]$.

However, after reconstruction using an eLDMC flap, the volume of the reconstructed breast can decrease due to postoperative atrophy of the LD muscle. In addition, it is difficult to adjust the symmetry of both breasts because the atrophy of the $\mathrm{LD}$ is further increased by severing the motor nerve of the $\mathrm{LD}$ during surgery in order to prevent synkinetic movement. Furthermore, few objective studies have investigated atrophy of the LD, and studies on the reduction of the volume of the LD after PORTx are rare. Extensive research has already been conducted on the effects of radiation therapy on normal tissues, and it is known to affect subcutaneous tissue, fatty tissue, muscle, and other tissue types [10]. Therefore, we investigated the magnitude of the volume reduction of the LD muscle after PORTx.

In a previous study, we found that the extent of atrophy of the LD was about $54 \%$ in patients who did not undergo postoperative chemotherapy or radiotherapy [11]. If there is a large amount of $\mathrm{LD}$ muscle in a flap, there may be more reduction of the breast volume due to $\mathrm{LD}$ atrophy. Therefore, if the $\mathrm{LD}$ itself is thick or the proportion of the LD in the flap is high, it is preferable to harvest slightly more than the expected amount. After radiotherapy, if the reconstructed breast is larger than expected, the volume may be easily reduced under local anesthesia. However, if the reconstructed breast is smaller than expected, correction of the breast is more complicated, as it would be necessary to use an implant under general anesthesia. Therefore, it is better to reconstruct the affected breast to be larger than the contralateral side.

In 28 patients, all slices in which the LD was found in the axial view of the CT were measured using the PACS system. The measurements were verified with the help of the Department of Radiology. However, a long period was necessary to collect the 28 subjects of this study because radiotherapy without chemotherapy after BCS was rarely performed, three breast surgeons interpreted the CT scans immediately after surgery and during patients' follow-up, and the timing of imaging was not consistent. In order to confirm the clinical outcomes, the patients were followed up for a sufficient period. As another step to reduce bias, only patients who had undergone serial CT scans at roughly the same intervals (7-10 days after the operation and $18 \pm 4$ months after the end of PORTx) were included.

The change in $\mathrm{LD}$ volume on CT scans after the end of PORTx was statistically significant $(\mathrm{P}<0.05)$, with decreases ranging from $61.19 \%$ to $80.82 \%$ in the $\mathrm{LD}$ volume compared to the CT scans taken 7 to 10 days postoperatively (Table 1 ). In the previous study of the authors [11], the LD volume reduction was roughly $15 \%$ greater in patients who were not treated with chemotherapy or radiotherapy, compared with a reduction of roughly $54 \%$. Therefore, if PORTx is planned, the volume of the $\mathrm{LD}$ should be reduced by $15 \%$. The reconstructed breast in the operating room should be larger than the contralateral breast, and it can be clinically confirmed that both breasts gradually become similar in size due to atrophy of the LD in the reconstructed breast.

Clinically, over the course of more than 3 years of follow-up, 
both the surgeon and patient were satisfied in 26 of 28 cases. One patient wanted postoperative bilateral breast enlargement, using a $165-\mathrm{mL}$ anatomical implant for the reconstructed breast and a $180-\mathrm{mL}$ anatomical implant for the opposite breast. One patient underwent additional reconstruction using implants for a severe breast deformity. However, even after the additional reconstruction, some of the deformities remained, leaving a difference in shape from the opposite breast. She did not want additional correction.

In conclusion, immediate breast reconstruction with an eLDMC flap at the same time as partial resection of the breast should be performed with a larger flap, based on a consideration of the volume reduction of the LD after PORTx. This study shows that when using an eLDMC flap with excised motor nerves of the LD muscle, the volume of the LD muscle was reduced by an average of $69 \%$ in patients receiving only PORTx. Special care should be taken when the LD in the eLDMC flap is thick or the LD occupies a large proportion of the flap.

\section{NOTES}

\section{Conflict of interest}

No potential conflict of interest relevant to this article was reported.

\section{Ethical approval}

The study was approved by the Institutional Review Board of Pusan National University Yangsan Hospital (IRB No. 05-2019021) and performed in accordance with the principles of the Declaration of Helsinki. Written informed consents were obtained.

\section{Patient consent}

The patients provided written informed consent for the publication and the use of their images.

\section{Author contribution}

Conceptualization: Park TS, Song KH, Nam SB. Data curation: Park TS, Seo JY, Nam SB. Formal analysis: Kim MW, Choo KS, Nam SB. Methodology: Seo JY, Choo KS. Project administration: Park TS. Visualization: Park TS, Kim MW, Lee JW, Jung YK, Choo KS. Writing - original draft: Choi JS, Song KH. Writing - review \& editing: Seo JY, Razzokov AS, Choi JS, Kim MW, Lee JW, Kim HY, Jung YJ.

\section{ORCID}

Tae Seo Park https://orcid.org/0000-0002-1491-0828 Jung Yeol Seo
Anvar S. Razzokov https://orcid.org/0000-0002-3886-0271

June Seok Choi https://orcid.org/0000-0003-2140-8550

Min Wook Kim https://orcid.org/0000-0001-8024-3608

Jae Woo Lee

Hyun Yeol Kim

Youn Joo Jung

Ki Seok Choo

Kyeong Ho Song

Su Bong Nam https://orcid.org/0000-0002-0945-6966 https://orcid.org/0000-0001-9008-1278 https://orcid.org/0000-0002-1311-4950 https://orcid.org/0000-0001-5072-4259 https://orcid.org/0000-0002-3419-972X https://orcid.org/0000-0002-9661-0879

\section{REFERENCES}

1. Fatah F. Extended latissimus dorsi flap in breast reconstruction. Oper Tech Plast Reconstr Surg 1999;6:38-49.

2. Menke H, Erkens M, Olbrisch RR. Evolving concepts in breast reconstruction with latissimus dorsi flaps: results and follow-up of 121 consecutive patients. Ann Plast Surg 2001; 47:107-14.

3. Chang DW, Youssef A, Cha S, et al. Autologous breast reconstruction with the extended latissimus dorsi flap. Plast Reconstr Surg 2002;110:751-9.

4. National Cancer Information Center. Cancer statistics [Internet]. Goyang: Ministry of Health and Welfare [cited 2018 Feb 13]. Available from: https://www.cancer.go.kr/

5. Kang SY, Kim YS, Kim Z, et al. Basic findings regarding breast cancer in Korea in 2015: data from a Breast Cancer Registry.J Breast Cancer 2018;21:1-10.

6. Clough KB, Cuminet J, Fitoussi A, et al. Cosmetic sequelae after conservative treatment for breast cancer: classification and results of surgical correction. Ann Plast Surg 1998;41: 471-81.

7. Gart MS, Smetona JT, Hanwright PJ, et al. Autologous options for postmastectomy breast reconstruction: a comparison of outcomes based on the American College of Surgeons National Surgical Quality Improvement Program. J Am Coll Surg 2013;216:229-38.

8. Maxwell GP. Iginio Tansini and the origin of the latissimus dorsi musculocutaneous flap. Plast Reconstr Surg 1980;65: 686-92.

9. Olivari N. The latissimus flap. Br J Plast Surg 1976;29:126-8.

10. Stone HB, Coleman CN, Anscher MS, et al. Effects of radiation on normal tissue: consequences and mechanisms. Lancet Oncol 2003;4:529-36.

11. Nam SB, Oh HC, Choi JY, et al. Volumetric change of the latissimus dorsi muscle after immediate breast reconstruction with an extended latissimus dorsi musculocutaneous flap. Arch Plast Surg 2019;46:135-9. 